\title{
PROPUESTA DE UN SISTEMA DE APOYO PARA LA ENSEÑANZA DE GEOMETRÍA EN LA CARRERA DE INGENIERÍA CIVIL
}

\author{
PROPOSAL FOR A SUPPORT SYSTEM FOR THE TEACHING OF GEOMETRY IN \\ THE CIVIL ENGINEERING CAREER
}

\section{NANCY VARELA TERREROS}

Universidad Católica de Santiago de Guayaquil. nancy.varela@cu.ucsg.edu.ec

RESUMEN

El artículo presenta la propuesta de un sistema de apoyo para el dictado de la Geometría en la carrera de Ingeniería Civil. A partir de encuestas a docentes y estudiantes, se encontró que la falta de visualización y la falencia en conocimientos de Matemáticas Básicas son las principales causas del bajo rendimiento histórico en esta asignatura. Dada la naturaleza de nativo digital del estudiante, y a la evolución del proceso enseñanza - aprendizaje a nivel global, la propuesta de apoyo se basa en el uso de las TIC.

PALABRAS CLAVES: geometría, TIC, visualización, sistema.
ABSTRACT The article presents the proposal of a support system for the dictation of Geometry in the Civil Engineering degree. From surveys of teachers and students, it was found that the lack of visualization and the deficiency in knowledge of Basic Mathematics are the main causes of the low historical performance in this subject. Given the nature of the student's digital native, and the evolution of the teaching-learning process at a global level, the support proposal is based on the use of ICT.

KEYWORDS: geometry, ICT, visualization, system. 


\section{INTRODUCCIÓN}

La asignatura de Geometría se encuentra en la base de la enseñanza de la Ingeniería Civil en la Universidad Católica de Santiago de Guayaquil, y sus contenidos programáticos están vinculados con asignaturas de todos los niveles. De aquí la importancia de un sistema que logre optimizar el desempeño de los estudiantes durante su tránsito por este curso.

Como parte de un trabajo de investigación a nivel doctoral, la autora propone el diseño de un sistema de apoyo con el uso de las TIC que contribuya a la mejora del rendimiento académico de los estudiantes en la asignatura de Geometría.

Un referente teórico considerado ampliamente como parte del diseño de este sistema de apoyo, lo constituye el Modelo Van Hiele para la enseñanza de la Geometría, que fue propuesto en 1957 por el matrimonio holandés Dina van Hiele-Geldof y Pierre van Hiele, quienes señalan que la enseñanza de la Geometría se construye pasando por diferentes niveles de pensamiento, siendo el primero de estos el de visualización o reconocimiento. Pese a los años transcurridos desde su creación, este modelo sigue vigente y ha sido considerado por diversos autores actuales. Así, la propuesta enfatiza la visualización como punto de partida de la enseñanza de la Geometría.

Para el diseño del sistema de apoyo se han considerado cinco elementos: herramienta de diagnóstico inicial, rúbricas, sistema de tutoría dual, libro electrónico (e-book), y pautas para el docente relacionadas con el uso del sistema. Todos estos elementos funcionan de manera coordinada, sin que sea recomendable manejar ninguno de ellos por separado. Como un eje transversal a su diseño, las Tecnologías de Información y Comunicación, TIC, juegan un papel determinante en la formulación de la propuesta.

DESARROLLO

Como parte de esta investigación se realizó un diagnóstico inicial de la situación de la enseñanza de Geometría en la carrera de Ingeniería Civil, a partir de 3 fuentes de información:

- Registro de calificaciones entre los semestres B2005 y B2016, en los que existe un promedio de $56.82 \%$ de reprobados en un total de 44 paralelos dictados.

- Encuestas a estudiantes, de cuyas respuestas se desprendió que:
- Una mayor visualización ayudaría a mejorar el rendimiento en la materia

- Esta visualización se daría en mejor forma con el uso de TIC

- Existen también factores externos a la UCSG, que le impiden estudiar de forma productiva

- Se deben brindar al docente nuevos recursos para el dictado de su clase

- Entrevista a docentes de Geometría, en la carrera de Ingeniería Civil - UCSG y otras universidades en Guayaquil y Samborondón. Las principales coincidencias entre los puntos de vista de los docentes fueron:

- El nivel académico alcanzado por el estudiante en el colegio, incide ampliamente en su rendimiento en el primer ciclo de la universidad, concretamente en la asignatura de Geometría.

- La visualización espacial es una de las competencias que más soporte necesita, puesto que de ellas depende la asimilación de los conceptos expuestos en la clase.

- La realización de ejercicios aplicativos constituye una acción de refuerzo tanto a nivel de trabajo autónomo individual, como a nivel de trabajo grupal con la debida supervisión, así como en su condición de herramienta para la evaluación.

- Las TIC sirven como herramienta para la mejor comprensión de la clase, porque optimizan la visualización de los conceptos expuestos.

- La formación del docente es fundamental para el éxito del curso de Geometría.

- Los factores propios del estudiante inciden de manera definitiva en el éxito en el curso de Geometría y en las demás asignaturas.

Al cotejar estas tres fuentes de información de diagnóstico, la postura de la autora es que el nivel elevado de reprobados se debe de manera principal a la falta de visualización, destacada por docentes y estudiantes, y al nivel de aprendizaje de Matemáticas Básicas alcanzado en el colegio. Por la naturaleza de nativo digital del estudiante, y de acuerdo con la evolución global del proceso enseñanza-aprendizaje, la propuesta de solución debe basarse en el uso de las TIC. 


\section{LA VISUALIZACIÓN Y EL MODELO DE VAN HIELE PARA LA ENSEÑANZA DE GEOMETRÍA}

Dina van Hiele-Geldof y Pierre van Hiele (1957), proponen un modelo sobre la enseñanza de la Geometría que se construye pasando por diferentes niveles de pensamiento, que son: $\mathrm{Re}^{-}$ conocimiento o Visualización, Análisis, Deducción informal u Orden, Deducción y Rigor. Este modelo, es mencionado por autores actuales como Crowley (1987), Feza y Webb (2005), Groth (2005), Vargas y Gamboa (2013), Haviger y Vojkujkova (2014), entre otros. Debido a la vigencia del uso del modelo, y a que inicia su análisis en la visualización del objeto geométrico en estudio, la autora acepta la aplicabilidad del modelo para la propuesta objeto de este estudio.

La presente investigación acepta la definición de visualización sugerida por Arcavi (2003), quien a partir de Zimmermann y Cunningham (1991) y Hershkowitz et al. (1989) da su propia definición de visualización y señala que: "Visualización es la capacidad, el proceso y el producto de la creación, interpretación, uso y reflexión sobre figuras, imágenes, diagramas, en nuestra mente, sobre el papel o con herramientas tecnológicas con el propósito de representar y comunicar información, pensar y desarrollar ideas previamente desconocidas y avanzar la comprensión” (Arcavi, 2003, p. 217).

La autora desglosa esta definición en algunos puntos de interés:

- Capacidad, proceso y producto - en un análisis por género próximo y diferencia específica, el autor no centra la definición de visualización tan solo en la "capacidad", como podría leerse en un diccionario, sino que genera una definición comprehensiva que abarca lo que se puede hacer con esta capacidad (proceso) y el resultado de la aplicación del proceso (producto).

- Creación, interpretación, uso y reflexión - la visualización, va más allá del sencillo paso de "observar". Gracias a la "capacidad", el objeto a visualizar se somete a estos "procesos" para generar un "producto".

- Sobre figuras, imágenes, diagramas - son el objeto a ser visualizado, como parte del análisis matemático. En el caso particular de la Ceometría, se refiere a los conceptos geométricos que ocupan un lugar en el espacio y como tales pueden ser medidos.

- En la mente, papel o con herramientas tecnológicas - el criterio de la autora es que estos tres escenarios no se excluyen entre sí. La mente es la protagonista, pero puede actuar sola o con cualquiera de los dos instrumentos señalados.

- Con el propósito de representar y comunicar información, pensar y desarrollar ideas previamente desconocidas. Si esta definición está realizada en el contexto de un proceso enseñanza - aprendizaje, entonces es válido recalcar este propósito en sus dos contextos:

- en la representación y comunicación de información, ya aceptada y analizada como parte de la "creación, interpretación, uso y reflexión" anteriores

- en el pensamiento y desarrollo de ideas previamente desconocidas, que fueron obtenidas a partir de la "creación, interpretación, uso y reflexión” anteriores

- Y avanzar la comprensión - lo que es su objetivo fundamental, como punto inicial para el proceso de enseñanza - aprendizaje.

LAS TIC EN LA ENSEÑANZA DE LA GEOMETRÍA

En relación con el uso de las TIC, cabe señalar que los estudiantes que ingresan a la universidad tienen un promedio de edad entre 17 y 18 años. Son nativos digitales y durante su educación media utilizaron a la computadora como un recurso para la ejecución de sus tareas, y para buscar información a través de la Internet. Para ellos, la Internet es una herramienta que no estuvo a su alcance únicamente desde una computadora sino desde su teléfono celular, su tablet, y actualmente, desde el propio televisor. A este respecto, en un estudio realizado sobre 716 escuelas españolas, Sigalés et al. (2009) concluyeron que "el $87.7 \%$ de alumnos tiene al menos un ordenador en su hogar y un $70.3 \%$ dispone de conexión a Internet" (p. 134). Aranguren (2015) al referirse a las TIC, generaliza diciendo que son "el conjunto de tecnologías desarrolladas para gestionar información y enviarla de un lugar a otro", y que los estudiantes de hoy tienen "habilidades naturales para la destreza de nuevas tecnologías y estas les ayudan a tener más información actualizada y de esta manera, desarrollan habilidades de búsqueda de información más rápido" (p.10). García et al. (2007), al referirse a los nativos digitales señalan que son una generación que: "ha crecido inmersa en las Nuevas Tecnologías, desarrollándose entre equipos informáticos, videoconsolas y todo tipo de artilugios digitales, convirtiéndose los teléfonos móviles, los videojuegos, Internet, el email y la mensajería 
instantánea en parte integral de sus vidas y en su realidad tecnológica" (p.2).

La autora observa que, siendo la computadora un instrumento, contribuye al proceso enseñanza - aprendizaje constructivista, cuando permite al estudiante construir sus propias transformaciones, mediante el uso de un software apropiado. En este sentido, la Ceometría tiene en las TIC una herramienta útil y necesaria que permite al estudiante:

- obtener y transmitir información global, en tiempo real, mediante el uso de la Internet,

- mejorar su proceso de visualización a través de la creación y/o recepción de imágenes mediante software especializado,

- construir su propio conocimiento mientras se desafía a sí mismo midiendo su aprendizaje mediante el uso de las TIC.

Con este antecedente, la propuesta para la mejora del aprendizaje de Geometría en la carrera de Ingeniería Civil - UCSG, ha de contemplar mejoras en la visualización y en los conocimientos de Matemáticas Básicas del estudiante, y ha de basar su funcionamiento en el uso de las TIC.

RESULTADOS

Para resolver el problema del bajo rendimiento histórico de los estudiantes de Geometría de la carrera de Ingeniería Civil UCSG, esta investigación propone un Sistema de Apoyo basado en las TIC para la enseñanza de Geometría. Para esta propuesta se recogen diversas definiciones de "Sistema", entre ellas, la brindada por Bunge (2004) quien señala que un sistema es un ente compuesto por cuatro elementos básicos: composición, entorno, estructura y mecanismo, y señala que toda cosa concreta es un sistema o un conjunto de sistemas. Quintana (2016), indica que "los sistemas se caracterizan por tener propiedades que sus componentes no poseen, a esto se lo reconoce como propiedades globales o emergentes" (p. 78). También se recoge la definición de sistema dada por Arnold y Osorio (1998), quienes dicen que los sistemas son "conjuntos de elementos que guardan estrechas relaciones entre sí, que mantienen al sistema directa o indirectamente unido de modo más o menos estable y cuyo comportamiento global persigue, normalmente, algún tipo de objetivo" (p. 3). Estas definiciones tienen en común que los elementos de un sistema guardan relación entre sí. Para la elaboración de la propuesta la autora defiende que un sistema es un conjunto de procedimientos - en este caso, procedimientos de apoyo - que logran a nivel interdependiente, mucho más que la suma de sus resultados independientes.

Este sistema consta de los siguientes componentes:

- Herramienta de diagnóstico inicial

- Sistema de tutoría dual que acompañe al estudiante durante su tránsito por el curso de Geometría

- Recurso educativo (e-book) que potencie la visualización de la geometría

- Pautas metodológicas que acompañen al docente en la implementación del sistema de acciones

- Rúbricas para la evaluación del aprendizaje en el curso de Geometría

\section{HERRAMIENTA DE DIAGNÓSTICO INICIAL}

Este componente está constituido por una evaluación, de modo presencial, que se realizará durante la primera semana del curso de Geometría. La herramienta tiene como objetivo segmentar al curso en dos grupos: aquellos que poseen conocimientos suficientes de Matemáticas Básicas para tener éxito en el desarrollo del curso, y aquellos que no los poseen.

Esta herramienta consiste de un grupo de problemas sobre los temas generales de Matemáticas en educación media. Los estudiantes que obtengan una calificación mínima de 7/10 serán considerados aptos, y no recibirán ningún soporte posterior. Los estudiantes que no alcancen esta nota mínima, serán direccionados al sistema de tutoría dual.

\section{SISTEMA DE TUTORÍA DUAL}

La aplicación del sistema de tutoría dual se realizará en la modalidad on-line, y se basará en la utilización de la plataforma informática de la UCSG. Esta plataforma permite al docente estar en contacto virtual con los estudiantes de una determinada asignatura, y generar con ellos actividades comunes tales como foros o talleres de trabajo, así como enviar y recibir archivos. En este sentido, la plataforma informática es una TIC en sí misma, que además permite el manejo de otras TIC, entre ellas, videos que facilitan el proceso de visualización que se pretende alcanzar en el estudiante.

Otra funcionalidad de la plataforma informática es la posibilidad de tomar lecciones en la modadlidad on-line. Dado que esta herramienta es de soporte, las calificaciones de estas 
evaluaciones no son vinculantes, y tienen como objetivo dar a conocer al estudiante su nivel de desempeño.

Tras obtener los resultados de la herramienta de diagnóstico inicial, el sistema de tutoría dual es aplicado a dos grupos diferentes de estudiantes:

- Aquellos que no obtuvieron una nota igual o superior a 7/10 en la herramienta de diagnóstico inicial - este grupo recibe una tutoría de soporte

- La totalidad de estudiantes del curso - este grupo, que incluye al anterior, recibe una tutoría general.

\section{SISTEMA DE TUTORÍA DE SOPORTE}

El primer grupo de estudiantes participa en una herramienta de tutoría de soporte, que solamente tendrá lugar durante el primer parcial. El detalle del sistema tutorial de soporte busca acompañar al estudiante para que pueda alcanzar los conocimientos iniciales de Matemáticas Básicas requeridos para mejorar su desempeño en la asignatura de Geometría.

El sistema se basa en la utilización de ejercicios de Matemáticas Básicas, tomados de libros de uso común en la enseñanza media, y en evaluaciones semanales de prueba. El docente publica semanalmente en la plataforma el tema correspondiente a la unidad que se desea revisar: uno teórico y uno con ejercicios propuestos. A partir de la tercera semana de clases, inicia la evaluación en línea, a través de la plataforma informática.

\section{SISTEMA DE TUTORÍA GENERAL}

Este sistema de tutoría se aplica a la totalidad de los estudiantes. Tiene una duración de quince semanas, iniciando luego de la aplicación de la herramienta de diagnóstico inicial. Se basa en la utilización de la plataforma informática de la UCSC y en el e-book que forma parte de esta propuesta.

En la ejecución de este componente, el docente publica semanalmente en la plataforma informática los soportes para el tema que se va a revisar. Dado que el e-book ha sido diseñado para trabajar de manera coordinada con esta tutoría, los soportes publicados en la plataforma se desglosan en:

- Bibliografía y ejercicios propuestos

- Un vínculo hacia una explicación en video, imagen o texto
- El capítulo correspondiente del e-book

Al igual que en la tutoría de soporte, a partir de la tercera semana el docente generará una evaluación en línea que se manejará desde la plataforma informática universitaria. A lo largo de todo el curso, se mantiene abierto un foro para la publicación de dudas por parte de los estudiantes.

En este sistema tutorial, el uso de las TIC se evidencia mediante:

- El manejo de la plataforma para evaluaciones, envío de archivos y foro

- El e-book, que contiene gráficos bidimensionales realizados en programas de diseño asistido por computadora, e hipervínculos al programa sketchup para visualización tridimensional

- El uso de la web a través del vínculo propuesto para la explicación en video, imagen o texto

- Como recurso áulico, mediante el empleo de la Internet para la visualización de videos y animaciones según los enlaces propuestos.

LIBRO (E-BOOK) BASADO EN LAS TIC

Como parte de la propuesta se ha diseñado un libro electrónico en formato PDF que cubre cada uno de los capítulos del curso de Geometría. El libro presenta la solución matemática y gráfica de los problemas que forman parte de la tutoría. Resuelve paso a paso cada uno de ellos, empezando por la definición de la incógnita y los pasos matemáticos que deben realizarse para su despeje.

Paralelamente, presenta gráficos que acompañan a estos pasos, facilitando así la comprensión del estudiante. Estos gráficos son de dos tipos:

- Bidimensionales, realizados en AutoCAD y luego insertados en el texto correspondiente

- Tridimensionales, realizados en SketchUp, y luego conectados al texto mediante un hipervínculo. Se escogió este programa por ser de libre descarga desde la web.

El e-book está diseñado para ser parte del acompañamiento al docente en el despliegue de la tutoría de soporte. También puede ser utilizado como recurso áulico, conforme las unidades que se manejan a lo largo del curso. 


\section{PAUTAS METODOLÓGICAS PARA ACOMPAÑAR AL DOCENTE EN LA IMPLEMENTACIÓN DEL SISTEMA DE \\ ACCIONES}

Las pautas metodológicas tienen como objetivo familiarizar al docente con el sistema de apoyo propuesto, definiendo las situaciones en que se debe usar y explicando cómo sus componentes son interdependientes.

Las pautas ayudan al docente a dar soporte al estudiante para avanzar de una fase a otra, según lo sugerido por el modelo de Van Hiele, dentro de los niveles de aprendizaje de Geometría. Estas fases son información, orientación dirigida, explicitación, orientación libre e integración.

La herramienta de diagnóstico inicial le sirve al docente en la fase de información para conocer el nivel de razonamiento y conocimiento que tiene el estudiante al iniciar su estudio de Geometría, mientras evalúa sus competencias iniciales. Con ayuda de las rúbricas, y a lo largo de la tutoría de soporte, puede conocer cómo evoluciona este conocimiento y razonamiento. La tutoría virtual ayuda al docente, conjuntamente con el e-book, para la orientación dirigida, cuando el estudiante es guiado a través de actividades y problemas para entender relaciones entre los diferentes elementos que conforman el objeto de estudio que debe visualizar. El docente, a lo largo del componente tutorial, puede orientar al estudiante para encontrar la solución a los problemas propuestos.

En la fase de la explicitación, el foro que se utiliza como parte de la aplicación del sistema tutorial, así como la propia aula de clases, permite discutir con el estudiante el aprendizaje obtenido en las fases anteriores, y expresarlo en lenguaje matemático. Consolidándose de esta forma las fases ya indicadas, el estudiante puede avanzar a los niveles siguientes, mediante orientación libre. Esto se evidencia con la ayuda de las tutorías, y el recurso educativo entregable e-book, que permiten al estudiante el aprendizaje autónomo.

Las evaluaciones no vinculantes y los talleres dirigidos que forman parte del sistema tutorial, con la ayuda de las rúbricas, permiten al docente detectar el nivel de conocimiento alcanzado por el estudiante, y llegar así a una fase de integración con ayuda de más ejercicios propuestos para trabajo autónomo, que fortalecen los conocimientos adquiridos antes de avanzar a conocimientos nuevos.

\section{RÚBRICAS PARA LA EVALUACIÓN DEL APRENDIZAJE EN EL CURSO DE GEOMETRÍA}

Para la elaboración de la propuesta, se recogen los puntos de vista de Varela (2017) quien a partir de Alsina et al. (2013), Gattica-Lara y UribarrenBerrueta (2012), y Torres y Perero (2010) señala que "una definición (...) empezaría por decir que una rúbrica es una herramienta de evaluación basada en criterios. La selección de los criterios de evaluación, así como la forma en que estos criterios serán evaluados, es el punto de inicio para el uso de la rúbrica” (p.5624). De acuerdo con lo analizado, siendo la Geometría una materia inicial, la rúbrica puede ser usada en dos momentos durante el curso:

- Intermedio, para valorar la evaluación del aprendizaje continuo a lo largo del curso

- Final, para valorar la evaluación del aprendizaje general del curso

Para la realización de estas rúbricas se necesitan algunos componentes:

- Académico: los contenidos del programa de geometría (momento intermedio y final)

- Visualización espacial del estudiante al inicio del curso. La autora propone el modelo Van Hiele, según lo citado por Crowley (1987) por ser de uso común en el curso de Geometría. El nivel inicial con que llega el estudiante se puede observar a partir de una herramienta de diagnóstico.

- Utilización por parte del estudiante de los apoyos informáticos a su alcance, principalmente las nuevas tecnologías de información y comunicación, TIC.

La rúbrica inicial debe permitir al docente conocer de manera global el nivel de conocimientos de Matemáticas Básicas con el que llega el estudiante al curso de Geometría. A partir de ahí, puede re-direccionar el curso, de modo que se facilite el proceso de aprendizaje. Para las rúbricas correspondientes al momento medio y final, debe primero tenerse claridad sobre cuáles son las unidades temáticas que se pretende evaluar. Las rúbricas deben responder a las siguientes preguntas:

- ¿Cuáles son las unidades temáticas que generan mayor dificultad de aprendizaje?

- ¿Cómo el nivel de visualización afecta la comprensión de la materia? 
- ¿Cómo se puede avanzar de un nivel al siguiente, en cada criterio?

- ¿Cómo los criterios pueden complementarse / afectarse entre sí?

Contestando estas preguntas, la rúbrica se convierte no únicamente en una herramienta de valoración de la evaluación de aprendizaje sino también en un elemento de apoyo para la mejora de todo el dictado de la asignatura.

Estos elementos están diseñados para ser utilizados en su conjunto, y son interdependientes entre sí. Pese a que cada uno de ellos tiene su valor, y presenta resultados positivos por sí mismo, se evidencia aquí la definición dada para "sistema", en el sentido de que los componentes logran a nivel interdependiente, mucho más que la suma de sus resultados independientes. Este logro a nivel interdependiente no se da únicamente desde el resultado final, en este caso, el rendimiento académico de los estudiantes, sino también en la forma de retroalimentación y ajuste a partir de los resultados de cada uno de ellos.

\section{DISCUSIÓN}

Dado que el sistema, según la definición propuesta para este documento, está formado por procedimientos que logran a nivel interdependiente mucho más que la suma de sus resultados independientes, se espera que los resultados de cada uno de estos procedimientos retroalimente y ajuste a los demás.

En el caso de la herramienta inicial de diagnóstico, este procedimiento brinda al docente los elementos necesarios para ajustar:

- Los elementos de la acción tutorial de soporte, a fin de que cubra principalmente los temas que resulten más necesarios, de acuerdo con la evaluación

- La rúbrica, tanto en los parámetros que van a ser medidos así como en la periodicidad en que se debe aplicar

La retroalimentación proporcionada desde el sistema tutorial dual, debe analizarse en sus dos componentes:

El sistema tutorial de soporte

- Está vinculado directamente a la herramienta de diagnóstico inicial, y es retroalimentado por ella. Además permite su ajuste si durante su aplicación resultan temas, de interés para el dictado de la Geometría, que no estén previstos en la herramienta de diagnóstico.
- Puede alimentar al e-book, en el sentido de indicar si hay elementos de las Matemáticas Básicas que deberían ser incluidos.

- Brinda elementos de interés para la mejora continua de la rúbrica.

- El sistema de tutoría general, brindado a la totalidad de los estudiantes, retroalimenta y ajusta:

- A la herramienta de diagnóstico inicial cuando descubre elementos de las Matemáticas Básicas que deben ser revisados.

- Al e-book, generando propuestas de mejora.

- Al igual que el sistema de tutoria general, brinda información para la mejora continua de la rúbrica

El e-book recibe retroalimentación directa desde la tutoría general, que es su escenario esperado de aplicación. A su vez, durante la utilización de este instrumento el docente está en capacidad de:

- ajustar el sistema tutorial, según los requerimientos de los estudiantes

- conjuntamente con el sistema tutorial, brindar elementos para la mejora de la rúbrica.

En cuanto a la aplicación de la rúbrica, ésta es aplicada durante momentos determinados del curso. El resultado de esta aplicación brinda elementos para identificar puntos que no se hayan tomado en cuenta en la herramienta de diagnóstico inicial, y reforzar los puntos que se hayan incluido en el sistema tutorial. Dado que el sistema tutorial general tiene como uno de sus instrumentos al e-book, este también será ajustado de acuerdo con los temas que proponga la rúbrica.

Se observa así que todos los elementos interactúan y son interdependientes. Es a partir de esta interdependencia que se espera que mejore el alcance de los objetivos de los estudiantes al iniciar el curso de Geometría. Las pautas metodológicas del docente, finalmente, se deben ajustar periódicamente conforme varíen los elementos que conforman este sistema. Las interacciones entre los componentes observan en la Figura 1:

- La rúbrica da y recibe información desde la herramienta de diagnóstico inicial, el sistema de tutoría y el e-book

- La herramienta de diagnóstico retroalimenta al sistema tutorial 


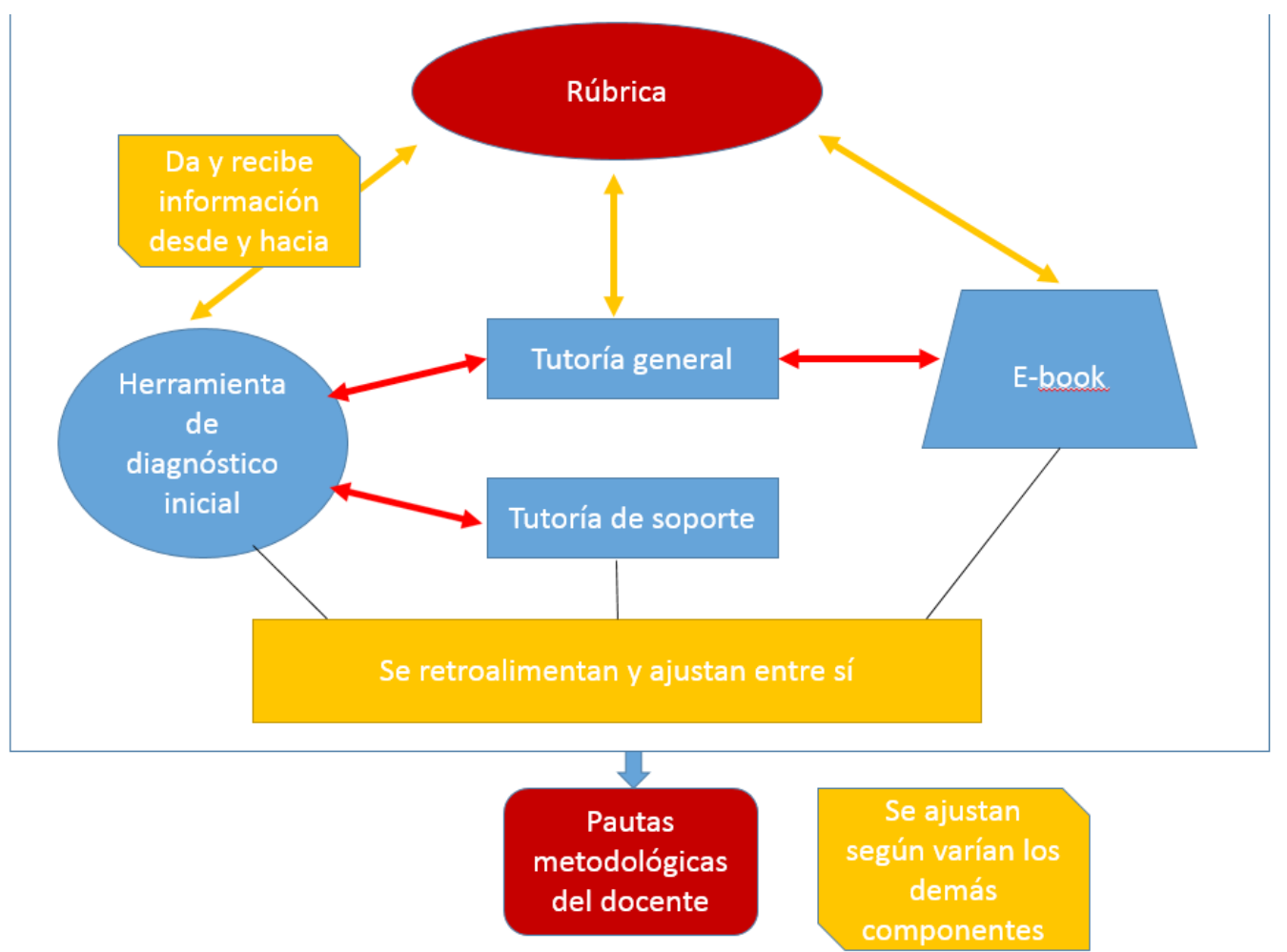

Fig. 1. Interacción de los componentes del sistema.

- El sistema tutorial y el e-book se retroalimentan entre sí

- Las pautas metodológicas se ajustan según varíen los demás componentes

De manera externa a la propuesta, dos componentes a considerar en el proceso de retroalimentación y ajuste son el desarrollo de la malla curricular y la evolución de las TIC.

\section{CONCLUSIONES}

El sistema de apoyo propuesto ha sido diseñado como soporte para el dictado de la asignatura de Geometría. Aún cuando, a la fecha de la elaboración de este documento, no ha sido implementado ni valorado, la autora arriesga las siguientes conclusiones:

- El sistema es replicable a otras asignaturas, y a otras unidades académicas. La metodología establecida en su elaboración permite generar pautas que pueden ser utilizadas en otros campos del conocimiento.

- La propuesta presentada es dinámica. Debe evolucionar, principalmente, de acuerdo con:

- la evolución de las TIC, en cuanto estas son parte del diseño de los componentes del sistema de apoyo.
- los requerimientos académicos exigidos durante la Educación Media, que marcan el campo de acción de la herramienta de diagnóstico inicial, y dan la pauta para las competencias iniciales del estudiante.

- los requerimientos académicos de la asignatura, que evolucionan conforme lo hace también la carrera de Ingeniería Civil, y que son los que determinan el contenido del sistema de apoyo.

- la normativa legal e institucional vigente, en cuanto ésta determine las pautas para el dictado de una asignatura.

\section{REFERENCIAS BIBLIOGRÁFICAS}

Alsina, J. et al (2013) Rúbricas para la evaluación de competencias. ICE y Ediciones OCTAEDRO S.L. Barcelona, España.

Aranguren, E. (2015) El uso de las TIC para el aprendizaje de Geometría. Universidad de Valladolid. Valladolid, España.

Arnold Cathalifaud, M.; Osorio, F. (1998) Introducción a los Conceptos Básicos de la Teoría General de Sistemas. Cinta de Moebio [en linea]. Disponible en: http://www.redalyc.org/ articulo.oa?id=10100306

Arcavi, A. (2003). The role of visual representations in the Learning of Mathematics. Educational Studies in Mathematics, Vol. 52, No. 3 (2003), pp. 215-241. Springer. 
Bunge, M. (2004) La investigación científica. Su estrategia y filosofía. Tercera edición. Siglo xxi Editores, S.A.de C.V. México D.F. México.

Crowley, M. (1987). The Van Hiele Model of the Development of Geometric Thought. Learning and Teaching Geometry, K-12, 1987 Yearbook of the National Council of Teachers of Mathematics, (1-16). Reston, Va.

Feza, N.; Webb, P. (2005) Assessment standards, Van Hiele levels, and grade seven learners' understandings of geometry. Pythagoras 62 . (36-47)

García, F.; Portillo, J.; Romo, J.; Benito, M. (2007) Nativos digitales y modelos de aprendizaje. Disponible en http://ceur-ws.org/Vol-318/ Garcia.pdf

Gatica-Lara, F.; Uribarren-Berrueta, T. (2013). ¿Cómo elaborar una rúbrica? Investigación en Educación Médica. Inv Ed Med 2013;2(1):6165. México D.F. Recuperado de http:// riem.facmed.unam.mx/sites/all/archivos/ V2Num01/10_PEM_GATICA.PDF

Groth, R. (2005) Linking theory and practice in teaching geometry. Mathematics teacher V. 99 (1) (27-30)

Haviger, J.; Vojkuvkova, I. (2015) The van Hiele levels at Czech secondary schools. Procedia Social and Behavioral Sciences. V 171 (2015) (977-981)

Hershkowitz, R. (1989) Visualization in Geometry - Two sides of the coin. Focus on Learning Problems in Mathematics. Winter Edition 1989. Volumen 11. Número 1. (61-74). Center for Teaching/Learning of Mathematics. Disponible en: https://www.researchgate.net/ publication/287200654_Visualization_in_geometry_two_sides_of_of_the_coin

Quintana, M. (2016) Sistema de rúbricas para la valoración de la evaluación del aprendizaje en la Universidad de Especialidades Espíritu Santo: experiencia en la carrera de Ingeniería en Sistemas. Universidad de La Habana. Editorial Universitaria. La Habana, Cuba.

Sigalés, C.; Mominó, J.; Meneses, J.; Badia, A. (2009) La integración de Internet en la educación escolar española: situación actual y perspectivas de futuro. Editorial Ariel, S.A. Barcelona, España.

Torres, J.; Perero, V. (2010). La rúbrica como instrumento pedagógico para la tutorización y evaluación de los aprendizajes en el foro online en educación superior. Revista de Medios y Educación. № 36 Enero 2010 pp.141 - 149. Sevilla. Recuperado de http://acdc.sav.us.es/ojs/ index.php/pixelbit/article/view/436/172

Varela, N. (2017). Rubrics as an alternative for the teaching of geometry at the engineering $\mathrm{fa}^{-}$ culty at the Catholic University of Santiago de Guayaquil. International Research Journal of Engineering and Technology (IRJET). Volumen 04 fascículo 06. Junio 2017. (5624-5627). Disponible en: https://www.irjet.net/archives/V4/ i6/IRJET-V4I6510.pdf

Vargas, G.; Gamboa, R. (2013) The van Hiele model and the teaching of the geometry. Uniciencia V. 27 (1) (74-94) 